\title{
Schickendantziella trichosepala (Alliaceae), nueva cita para Bolivia
}

\section{Schickendantziella trichosepala (Alliaceae), new record from Bolivia}

\author{
María A. Negritto ${ }^{1}$, Eduardo Ruiz ${ }^{1}$, Stephan Beck ${ }^{2}$, Inelia Escobar ${ }^{1}$ \& Carlos M. Baeza ${ }^{1}$ \\ ${ }^{1}$ Departamento de Botánica, Facultad de Ciencias Naturales y Oceanográficas, Universidad de Concepción, Casilla 160-C, \\ Concepción, Chile. \\ ${ }^{2}$ Herbario Nacional de Bolivia, Docente-Investigador del Instituto de Ecología, Universidad Mayor de San Andrés, La Paz, \\ Bolivia. \\ mnegritto@udec.cl
}

\begin{abstract}
Schickendantziella trichosepala (Speg.) Speg., a new record from Bolivian flora. The taxonomic history, description, iconography and geographical distribution of this monotypic South American genus are presented.
\end{abstract}

Schickendantziella fue descrito por Carlo Luigi Spegazzini en honor al distinguido químico D. F. Schickendantz como un nuevo género de Liliaceae. Originalmente, el nombre fue Schickendantzia, publicado en 1896 y transferido posteriormente en 1903 a Schickendantziella por el mismo Spegazzini debido a la prioridad delnombreSchickendantzia Pax (Alstroemeriaceae) publicado en 1889. Hutchinson (1939) lo reconoce dentro de las Amaryllidaceae, basado en la descripción del mismo Spegazzini. Actualmente, Schickendantziella, género monotípico, ha sido incluido en la tribu Gilliesieae Lindley, familia Alliaceae (Fay \& Chase 1996) tribu con distribución principalmente en la zona mediterránea de Chile.

Schickendantziella trichosepala (Speg.) Speg., Anales Mus. Nac. Buenos Aires. 3(2): 8. 1903.

Basónimo: Schickendantzia trichosepala Speg., Rev. Fac. Agron. Veter. 23-24: 386. 1896.

Tipo: Hab. In rupestribus, Quebrada de Colalao, Tucumán, Spegazzini 11165 (LP).

Actualmente, Katinas et al. (in prep.) elaboran un catálogo de los materiales tipos que fueron recolectados por Spegazzini. Se estima que sólo en el Herbario de La Plata, son aproximadamente 700 los materiales tipos de la colección de Spegazzini (Katinas et al. 2000).

Según Katinas (com. pers.), el ejemplar Spegazzini 11165 depositado en el herbario de La Plata (LP) correspondería al ejemplar tipo de esta especie. El protólogo menciona "In fissuris rupium muscosarum secus rivulus in sylvis Alnorum in Prov. Tucuman" y la etiqueta escrita por la mano del mismo Spegazzini señala "Schickendantziella trichosepala Speg., typus novi generis et species. Hab. in rupestribus, Quebrada de Colalao, Tucumán ". Si bien la fecha señalada en el ejemplar es posterior, 1898, ésta correspondería a la revisión del ejemplar con el nuevo nombre y no a la fecha de recolección del material, no habiendo duda de que se trata efectivamente del material tipo.

\section{DESCRIPCIÓN DEL TAXÓN}

Plantas herbáceas, bulbosas, inconspicuas. Bulbos ovalados a piriformes, de $1-2,5 \mathrm{~cm} \times 5-13 \mathrm{~mm}$, generalmente a $7 \mathrm{~cm}$ de profundidad. Hojas 2-5, lineares, de $10-20 \mathrm{~cm} \times 2-4 \mathrm{~mm}$, ápices obtusos. Escapos ascendentes o arqueados, de 10-30 cm x 1-1,5 mm, igual o más largos que las hojas, cilíndricos, glabros, terminados en 1 ó 2 flores. Brácteas enteras, de 1,52,5 cm x 2-3 mm, desiguales, lineares, agudas, membranáceas con bordes hialinos, la interior más corta. Pedicelos simples de 0,8-3(-5) cm, generalmente arqueados después de la antesis; tépalos externos, 3, de 1,4-3,2 cm x 1-2 mm, verdecremoso, de base triangular, unidos sólo en la base, con bordes pestañosos terminando en lacinias purpúreas. Tépalos internos ausentes. Androceo tubular, con 6 estambres fértiles, 3 más largos alternando con 3 más cortos, con filamentos soldados, formando un tubo que rodea el ovario, anteras introsas de 1-1,2 mm. Gineceo tricarpelar, trilocular, ovario súpero, cilíndrico de $8-10$ × 2-2,5 mm, estilo filiforme y estigma trífido, papiloso. Fruto desconocido (Fig. 1).

Hasta el año 1935, fecha de la última recolección, Schickendantziella trichosepala sólo se conocía para Argentina y las descripciones se han hecho sobre la base del escaso material existente en los herbarios CORD, LIL, LP y SI. Sin embargo, uno de los coautores recolectó en 1987, material correspondiente a esta especie, en el sur de Bolivia, constituyendo éste un nuevo registro para la flora de Bolivia. 

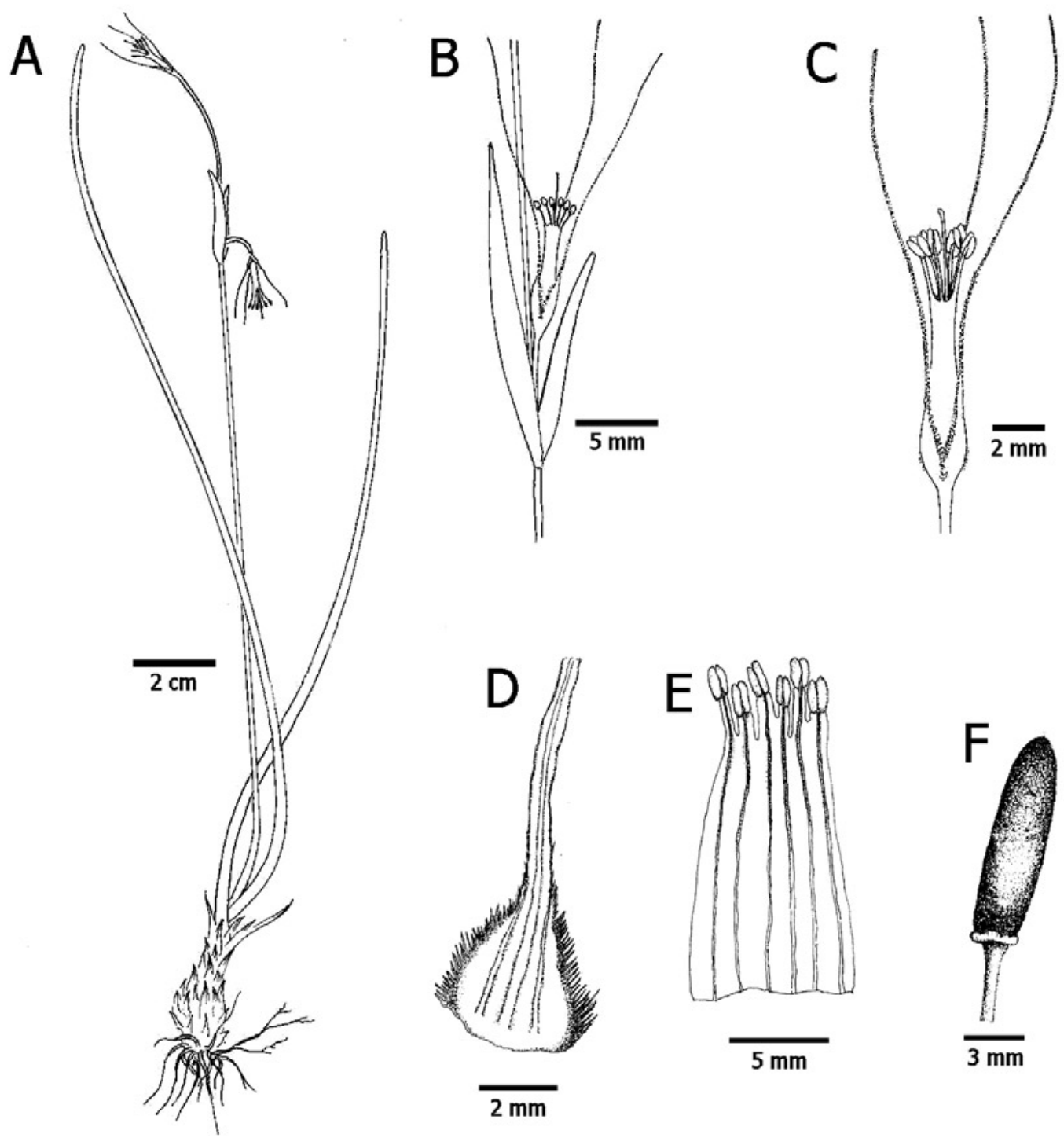

Figura 1. A. Planta, B. flor rodeada por brácteas, C. flor, D. tépalos, E. androceo hexámero, F. ovario.

Figure 1. A. Plant, B. flower with a pair of bracts, C. flower, D. tepals, E. androecium with two whorls of stamens, F. ovary.

DistRIBUCIÓN Y HÁBITAT: Schickendantziella trichosepala es una especie extremadamente rara que crece entre las grietas de las rocas en suelo humoso, en lugares sombríos, húmedos y calurosos, especialmente a lo largo de los arroyos en la selva tucumano-oranense, en bosques de alisos, Alnus acuminata Kunth (Spegazzini 1896a, 1896b). En Argentina, se la encuentra en las provincias de Jujuy, Salta y Tucumán (Hauman \& Vanderveken 1917, Zuloaga \& Morrone 1996, Zuloaga et al. 2008) y en Bolivia en Tarija, provincia de Arce.

Material examinado: BOliViA. Dpto. Tarija: Prov. Arce. Cerca Rejará. $500 \mathrm{~m}$ hacia el oeste del Abra Blanca.
Bosque abierto de Alnus acuminata sobre ladera de exposición sur a 2.940 m s.m. 17-XII-1987. S.G. Beck \& M. Liberman 16050 (LPB, US). ARGENTINA. Prov. Jujuy: Dpto. Tilcara, Cimarrones, Obispo, 16-I-1974, "flor violácea". Kiesling et al. 424 (LP). Dpto. Tumbaya, en Volcán, I-1910. Castillón 6756 (LIL 050803). Volcán, a 3.000 m s.m., 13-I-1919. Castillón 6707 (LIL 50906). Prov. de Tucumán. Valles Calchaquíes, Rincón de los Quilmes, 2.800 m s.m., 11-I-1913. Castillón 2476 (CORD, LIL 050905, SI). Valles Calchaquíes, El Molle, 29-I-1935. Castellanos s.n. (CORD). Valles Calchaquíes, Colalao, Managua Guacamayo, "bajo los arbustos”, 2.900 m s.m., 6-II-1917. Castillón 109b (LIL 30812). 


\section{AGRADECIMIENTOS}

Proyecto DIUC 208.111.049.1-0 (Universidad de Concepción). A Rosa Guaglianone, del Instituto de Botánica Darwinion, Ana María Figueroa del Herbario Lillo, Liliana Katinas, Laura Iharlegui y Diego Gutiérrez del Herbario de La Plata por su valiosísima colaboración. A los revisores por sus comentarios.

\section{BIBLIOGRAFÍA}

FAY, M.F. \& M.W. Chase. 1996. Resurrection of Themidaceae for the Brodiaea alliance, and recircumscription of Alliaceae, Amaryllidaceae and Agapanthoideae. Taxon 45: 441-451.

Hauman, L.L. \& G. Vanderveken. 1917. Catalogue des Phenérogames de 1'Argentine. Anales del Museo Nacional Historia Natural de Buenos Aires. 29: 271-272.

Hutchinson, J. 1939. The tribe Gilliesieae of Amaryllidaceae. Herbertia 6: 136-145.

Katinas, L., D. Gutiérrez \& S. Torres-Robles. 2000. Carlos Spegazzini (1858-1926): travels and botanical Works on vascular plants. Annals of the Missouri Botanical Garden
87: $183-202$.

PAX, F. 1889. Beiträge zur Kenntnis der Amaryllidaceae. Botanische Jahrbücher 11: 318-338.

Spegazzini, C. 1896a. Algunas observaciones sobre la flora de La Ventana. Revista Facultad de Agronomía y Veterinaria 23-24: 386

Spegazzini, C. 1896b. Plantae Novae V. Criticae, Reipublicae [sic] Argentinae, Decas III. Revista de la Facultad de Agronomía y Veterinaria, Universidad Nacional de La Plata, 2 (23-24): 382-392.

Spegazzini, C. 1903. Notes synonymiques. Anales del Museo Nacional de Historia Natural de Buenos Aires, Ser. 3, 9 (2): 7-9.

Zuloaga, F.O. \& O. Morrone (eds.). 1996. Catálogo de las plantas vasculares de la República Argentina. I. Pteridophyta, Gymnospermae y Angiospermae (Monocotyledoneae). Monograph in Systematic Botany from Missouri Botanical Garden 60: i-xviii, 1-323.

Zuloaga, F. O., O. Morrone \& M. Belgrano (eds). 2008. Catálogo de las Plantas Vasculares del Cono Sur (Argentina, Sur de Brasil, Chile, Paraguay y Uruguay). Pteridophyta, Gymnospermae, Monocotyledoneae. Monograph in Systematic Botany from Missouri Botanical Garden 107(1); i-xcvi, 1-983. 